\title{
Innovazione, qualità e marketing responsabile nel settore agroalimentare
}

\author{
Azzurra Annunziata, ${ }^{1}$ Irene P. Borrelli, ${ }^{1}$ Rosa Misso, ${ }^{1}$ Domenico Regazzi ${ }^{2}$ \\ 'Dipartimento di Studi Economici S. Vinci, Università di Napoli Parthenope, Fondazione Simone \\ Cesaretti; \\ ${ }^{2}$ Dipartimento di Economia e Ingegneria Agrarie, Alma Mater Studiorum Università di Bologna, \\ Italy
}

\begin{abstract}
The agri-food enterprises are more and more aware of their responsibilities and their social role and they require the development of innovative marketing strategies, based on the principles of sustainability and characterized by a strong orientation to the society. Starting from the importance of innovation for the sustainable development in socio-economic and environmental terms, the study analyzes the links between sustainable innovation and food quality focusing on the communication innovations. In this regard, the study provides an overview on the evolution of marketing theory (ethical, social, responsible and sustainable), underlining the need to push also the agri-food system towards a responsible valorization as an innovative form of sustainable communication. Finally, it provides useful insights to policy makers and stakeholders on the tools required to foster and support sustainable development of agri-food sector and to create synergies between public and private interests.
\end{abstract}

\section{Riassunto}

Correspondence: Domenico Regazzi,

Dipartimento di Economia e Ingegneria Agrarie, Alma Mater Studiorum Università di Bologna, Italy.

E-mail: domenico.regazzi@unibo.it

Key words: innovation, food quality, agri-food marketing, responsible valorization, social responsibility.

Parole chiave: innovazione, qualità alimentare, marketing agroalimentare, valorizzazione responsabile, responsabilità sociale.

Received for publication: 13 June 2011.

Accepted for publication: 17 October 2011.

Società rappresentata nell'ambito AISSA: Società Italiana di Economia AgroAlimentare - SIEA.

(C) Copyright A. Annunziata et al., 2011

Under no circumstances figures can be used without prior written consent of the copyright owner.

Licensee PAGEPress, Italy

Italian Journal of Agronomy 2011; 6(s2):e11

doi:10.4081/ija.2011.6.s2.e11

This work is licensed under a Creative Commons Attribution NonCommercial 3.0 License (CC BY-NC 3.0).
Le imprese agroalimentari, sempre più consapevoli delle loro responsabilità e del loro ruolo sociale, richiedono lo sviluppo di strategie di marketing innovative, basate sui principi di sostenibilità e caratterizzata da un forte orientamento alla società. Partendo dall'importanza dell'innovazione per il perseguimento di uno sviluppo sostenibile in termini socio-economici e ambientali, lo studio analizza i legami tra l'innovazione sostenibile e la qualità alimentare concentrandosi, infine, sulle innovazioni nel campo della comunicazione. Al riguardo, il lavore offre una panoramica sull'evoluzione della teoria di marketing (etico, sociale, responsabile e sostenibile), sottolineando la necessità di orientare anche il sistema agro-alimentare verso la valorizzazione responsabile, intesa come forma innovativa di comunicazione sostenibile. Infine, il lavoro intende fornire utili indicazioni ai policy maker ed agli stakeholder per promuovere e sostenere lo sviluppo sostenibile del settore agroalimentare e per la creazione di sinergie tra interessi pubblici e privati.

\section{Introduction}

Le crescenti preoccupazioni di ordine socio-economico ed ambientale attribuiscono alle imprese che operano nel settore agroalimentare un ruolo centrale nella realizzazione di un percorso di sviluppo orientato alla sostenibilità. Lo scenario di riferimento del settore va richiedendo, infatti, nuove risposte in funzione del rallentamento dei ritmi globali della crescita, dei sempre più numerosi casi di inquinamento ambientale, di depauperamento delle risorse naturali, così come delle preoccupazioni per la salute pubblica, sempre più interessata dall'incremento delle malattie croniche (che vedono tra i principali fattori di rischio l'obesità, l'abuso di alcol, cattive abitudini alimentari, stili di vita sedentari o l'ipertensione arteriosa). La salute, però, è messa a rischio anche da fattori ambientali che indirettamente possono, ad esempio, determinare una contaminazione dei prodotti alimentari. Ad ogni modo, questo nuovo scenario di riferimento, negli ultimi anni, sta determinando sempre di più un ripensamento dell'agire delle imprese in un'ottica di sostenibilità. In particolare, l'impresa agroalimentare è chiamata ad andare oltre se stessa, rivedendo le sue funzioni alla luce di un maggior orientamento verso la società, superando il ruolo di semplice agente economico per acquisire sempre più la funzione di traino per il benessere della società.

L'Unione Europea, al riguardo, suggerisce di guardare alle determinanti di questi nuovi orientamenti come a veri e propri motori di innovazione, in grado di innescare meccanismi di sviluppo sostenibile (Commissione Europea, 2008). Ciò, naturalmente, impone di guardare al ruolo innovatore dell'impresa, riconoscendo nelle loro responsabilità una condizione imprescindibile per l'acquisizione di un posizionamento competitivo sostenibile. Il processo evolutivo che attualmente sta interessando il settore agroalimentare, così, oggi vede l'innovazione come la principale leva per garantire uno sviluppo sostenibile del 
settore e nel suo sistema di riferimento. Così, ad esempio, se da un lato cresce l'interesse, a livello scientifico oltre che istituzionale e produttivo, per prodotti come ad esempio i functional foods che hanno lo scopo di ottimizzare il benessere fisico e ridurre il rischio di malattie, dall'altro, si accende sempre di più il dibattito sulle soluzioni possibili provenienti in maniera sempre più incisiva dalle moderne biotecnologie, dalle nanotecnologie o dall'impiego di Organismi Geneticamente Modificati (OGM). Tali interessi fanno sì che la produzione di beni e la prestazione di servizi siano tutti proiettati nella soddisfazione di valori, bisogni e desideri espressi dalla società e dai consumatori. Ciò è testimoniato dalla moltiplicazione a livello globale di azioni e progetti implementati dalle imprese per fornire un contributo ad una causa sociale 0 ambientale e che vedono coinvolte le diverse funzioni dell'impresa: dalla produzione alla distribuzione e al marketing fino alle numerose forme in cui l'impresa svolge il suo ruolo di agente territoriale.

Alla luce di tali considerazioni, il presente lavoro mira ad evidenziare come le evoluzioni del sistema agroalimentare stiano pretendendo sempre di più strategie di marketing agroalimentare innovative e ispirate ai principi di sostenibilità, connotate da un forte orientamento alla società. Partendo dall'importanza dell'innovazione per il perseguimento di uno sviluppo sostenibile in termini socio-economici ed ambientali, il lavoro mette in evidenza i legami esistenti tra innovazione sostenibile e qualità alimentare concentrandosi in particolare sulle innovazioni nel campo della comunicazione. Al riguardo, il lavoro offre una rassegna sulle evoluzioni teoriche del marketing (etico, sociale, responsabile e sostenibile) che sottolineano la necessità di integrare anche per il sistema agroalimentare le spinte verso una valorizzazione responsabile come forma innovativa di comunicazione sostenibile.

Inoltre, il lavoro intende fornire spunti di riflessione ai policy maker ed agli stakeholder sugli strumenti necessari per accompagnare e sostenere uno sviluppo sostenibile del settore agroalimentare e per creare sinergie tra interessi pubblici e privati.

\section{Innovazione, sviluppo sostenibile e agroalimentare}

\section{Il ruolo dell'innovazione}

Com'è ormai ampiamente riconosciuto, l'innovazione ${ }^{1}$ rappresenta uno dei fondamenti dello sviluppo, ma anche la principale causa del declino di sistemi economici, della performance di imprese, della competitività internazionale di industrie e di interi paesi. Fin dal lavoro pioniere di Joseph Schumpeter (Schumpeter, 1942;1947), l'innovazione ha rappresentato un tema ampiamente dibattuto in economia; eppure, ad oggi, non si è pervenuti ad una definizione univocamente utilizzata. Le riflessioni in merito sono state numerosissime, in funzione, soprattutto, della crescente importanza che l'innovazione ed il cambiamento tecnologico hanno acquisito nella storia del pensiero economico: si pensi, ad esempio ai contributi di Rosenberg (1991) oppure Freeman (1997), ma anche Adam Smith, David Ricardo e Karl Marx si erano già interrogati sul ruolo della tecnologia nell'economia (Malerba, 2000). Nel Libro Verde del 1995, la Commissione Europea definiva l'innovazione come il rinnovo e l'ampliamento della gamma dei prodotti e dei servizi, nonché, dei mercati ad esse associati; l'attuazione di nuovi metodi di produzione, di approvvigionamento e di distribuzione; l'introduzione di mutamenti nella gestione, nell'organizzazione e nelle condizioni di lavoro, nonché nelle qualifiche dei lavoratori (European Commission, 1995).

${ }^{1}$ Per Schumpeter l'innovazione consiste nel fare qualcosa di nuovo nel sistema economico, e ciò non richiede necessariamente un'invenzione corrispondente (Malerba F., 2000). In generale, egli defini l'innovazione come uno spostamento della funzione di produzione dovuto non solo ad innovazioni di prodotto o di processo ma anche all'apertura di nuovi mercati, all'acquisizione di una nuova fonte di materie prime o alla so ma anche all'apertura di nuovi mercati, all'acquisizione di una nuova fonte di materie prime o alla
ristrutturazione di un'azienda. Particolarmente interessante risulta, poi, il concetto d'innovazione proveniente da Porter: Linnovazione implica sovente idee che non sono neppure nuove, idee che hanno circolato ma che nessuno ha mai cercato seriamente di mettere in pratica (Porter, M. E., 2001).
Da allora, sotto l'egida della stessa Commissione, è stata avviata una politica europea dell'innovazione pervasiva, affinché nessun settore rinunciasse ai potenti vantaggi che essa sarebbe stata capace di generare in termini di competitività. ${ }^{2}$ Così, in occasione del Consiglio Europeo di Lisbona del 2000, la Commissione Europea battezzerà l'innovazione come uno degli obiettivi strategici a sostegno dello sviluppo futuro, mentre, la Presidenza del Consiglio Europeo di Bruxelles del 2005 ribadirà la necessità dell'Unione Europea di puntare sulla conoscenza, sull'innovazione e sulla valorizzazione del capitale umano per rinnovare le basi della sua competitività, aumentare il suo potenziale di crescita e la sua produttività e rafforzare la coesione sociale (http://www.consilium.europa.eu/ueDocs/cms_Data/docs/pressData/it/e c/84343.pdf).

Nel tempo, poi, l'interesse dell'Unione Europea per l'innovazione è andato crescendo, soprattutto, in funzione del progressivo acuirsi, nel mondo, della concorrenza per attirare investimenti in ricerca, sviluppo e, appunto, innovazione. Così, l'Unione ha finito con il porre la ricerca e l'innovazione al centro dell'attività economica, in quanto motori di crescita e sviluppo per qualsiasi settore di riferimento.

\section{Innovazione, agroalimentare e sostenibilità}

Impegnata nella costruzione dell'Europa del 2020, oggi l'Unione Europea definisce l'innovazione come il fattore chiave non solo per la creazione di una maggiore occupazione, per la costruzione di una società più verde e per il miglioramento della nostra qualità della vita, ma anche per il mantenimento della competitività sui mercati globali. ${ }^{3}$ In particolare, essa va sempre di più perseguendo l'innovazione sostenibile, ovvero, innovazioni di prodotto, di processo ed organizzative, in grado di assicurare la sostenibilità ambientale, sociale ed economica dei diversi settori che contribuiscono allo sviluppo della società. L'accezione dell'innovazione quale indirizzo privilegiato per lo sviluppo sostenibile dei settori economici rappresenta così un tema cruciale nelle moderne economie dell'informazione e della conoscenza (Foray, 2000). A fronte di tali impulsi il settore agroalimentare ha reagito con un impegno crescente delle imprese in una gestione sempre più competitiva delle informazioni che, secondo un approccio evoluzionista, testimonierebbe l'attivazione da parte del settore stesso di un processo innovatore che interessa il prodotto, il processo e l'organizzazione, mentre, la ricerca della competitività si è andata sempre più confrontando con l'obiettivo della sostenibilità socio-economica ed ambientale (Dosi, 1982; Westgren, 1998; Bowersox et al., 1980; Pick et al., 2001).

Ciò che più preme evidenziare nel presente lavoro è il crescente consolidamento del vincolo tra fenomeni innovativi, agroalimentare e sviluppo sostenibile. Tale legame, in verità, può essere analizzato da due differenti prospettive. Da un lato, il ricorso all'innovazione come fattore di animazione per la sostenibilità del settore agroalimentare viene perseguito per assicurare la sostenibilità socio-economica ed ambientale del settore stesso, dall'altro, per far sì che il settore contribuisca al mantenimento nel tempo e nello spazio delle condizioni economiche, sociali ed ambientali che determinano lo sviluppo umano nel complesso e, quindi, non solo lo sviluppo di uno specifico settore. L'accresciuta sensibilità dei consumatori ai temi della salute, dell'ambiente o dell'equità e la relativa ricerca di un miglioramento del benessere e della qualità di vita, hanno stimolato un interesse crescente verso il complesso di attività da intraprendere non solo al fine di orientare l'impresa sempre più verso la società ma soprattutto per generare una visione d'impresa strettamente connessa ad un impegno umanitario, sociale 0 ambientale.

${ }^{2}$ Nel 1996, il Primo Piano d'Azione per l'Innovazione in Europa, ha avviato il processo di costruzione di un quadro politico, normativo e finanziario che spronerà gli stati membri ad aumentare la quota del prodotto interno lordo destinato alla ricerca, allo sviluppo e all'innovazione.

${ }^{3}$ http:/lec.europa.ew/research/innovation-union/index_en.cfm 
Se da un lato tali orientamenti costituiscono dei veri e propri propulsori dell'innovazione, dall'altro, essi hanno determinato un'evoluzione del sistema agroalimentare che è andata sempre più pretendendo strategie di produzione, di logistica e distribuzione, così come di informazione e comunicazione innovative, ispirate ai principi di sostenibilità e connotate da un forte orientamento alla responsabilità.

Il processo evolutivo che attualmente sta interessando il settore agroalimentare, così, oggi vede l'innovazione come la principale leva per garantire uno sviluppo sostenibile del settore e nel suo sistema di riferimento. Innanzitutto, la stretta connessione tra competitività e innovazione, costituisce una garanzia per uno sviluppo economico sostenibile del settore agroalimentare in quanto permette di far perdurare nel tempo la capacità di acquisizione di vantaggi competitivi sia dei first mover che dei detentori di specifiche e nuove competenze e conoscenze (Knudson et al., 2004).

In termini sociali, la inclusività dell'innovazione, spinge poi ad una diffusione capillare delle innovazioni in campo agroalimentare sia tra le imprese (in quanto foriera di crescenti opportunità) che tra i consumatori (che possono beneficiare di una progressiva riduzione dei prezzi e di un minor costo per l'acquisizione delle informazioni sulla salute, sull'ambiente di produzione o sui territori di riferimento).

In termini ambientali, infine, il processo di greening avviato dalla Politica Agricola Comunitaria e sostenuto dalla progressiva integrazione delle politiche di ordine ambientale nello sviluppo del settore agricolo ed alimentare riconosce proprio nell'innovazione la chiave di volta per assicurare nel tempo e nello spazio l'integrità dei fattori naturali, delle risorse ambientali e paesaggistiche. Si tratta di fattori che si configurano come veri e propri beni primari per l'impresa agroalimentare e che arricchiscono di significato i relativi prodotti (Detre et al., 2011).

In tale ottica, l'innovazione, garantendo la diminuzione dei costi di produzione o dell'impatto ambientale e sociale, l'aumento della disponibilità di capitale nel lungo periodo come pure della quota di mercato, si pone come vero e proprio strumento per la sostenibilità dei processi interni ed esterni dell'impresa, delle sue relazioni con il mercato, con i consumatori e con la società nel suo complesso. In verità, relativamente al settore agroalimentare, l'applicazione del concetto di innovazione si presta a numerose interpretazioni. Interessando l'intero sistema, tali innovazioni sono il risultato di progressi scientifici in campo agro-biotecnologico (volti ad esempio ad incrementare le risorse alimentari globali offrendo però agli agricoltori opportunità di protezione e miglioramento dei raccolti unitamente ad un uso più efficiente delle risorse naturali), nel campo dell'alimentazione, in campo ambientale, ma anche nel campo della logistica e della comunicazione. Così dagli organismi geneticamente modificati agli alimenti funzionali si va all'agricoltura di precisione, allo sviluppo delle moderne tecnologie dell'informazione e della comunicazione nei diversi settori produttivi e, quindi, ad una visione del settore in costante evoluzione, o meglio, rivoluzione.

In tale contesto, si fa sempre più strada l'impresa innovatrice che rivede la propria filosofia di gestione alla luce di una nuova missione improntata sulla società e orientata al soddisfacimento di interessi generali comuni. La produzione di beni, la prestazione di servizi e lo sviluppo dell'innovazione, così, sono tutti proiettati nella soddisfazione di valori, bisogni e desideri espressi dalla società e dai consumatori. Si tratta in verità di un orientamento testimoniato dalla moltiplicazione a livello globale di azioni e progetti implementati dalle imprese per fornire un contributo ad una causa sociale, nell'ambito del perseguimento di un obiettivo pur sempre commerciale.

\section{Innovazione sostenibile e qualità alimentare}

Le crisi sanitarie e gli scandali alimentari, che negli ultimi anni hanno interessato il settore agro-alimentare, hanno inciso significativamente sull'opinione pubblica, creando una diffusa preoccupazione sulla qualità, salubrità e sulla sicurezza dei prodotti stessi. Al tempo stesso, lo stretto legame esistente fra salute e alimentazione, l'impatto dell'attività agricola sull'ambiente, la familiarità e interdipendenza dei prodotti con il territorio di produzione impongono agli operatori delle filiere agroalimentari, di valutare gli stimoli e le richieste provenienti dalla domanda, soprattutto in termini di maggiore eticità sia dei prodotti che delle strategie aziendali (Briamonte e Hinna, 2008).

Questi mutamenti di scenario, hanno posto gli attori delle filiere agroalimentari di fronte a nuove sfide commerciali imponendo il ricorso a strategie innovative capaci di sostenere la qualità dei prodotti offerti. ${ }^{4}$ Tuttavia, appare necessario che le produzioni agroalimentari di qualità siano in grado di garantire non solo una equa remunerazione agli operatori del settore e la soddisfazione delle esigenze espresse dai clienti/consumatori, ma anche assicurare una gestione sostenibile dei giacimenti ambientali, intesi come risorse naturali e paesaggistiche; concorrere al miglioramento della salute collettiva e all'incremento della qualità della vita; contribuire al mantenimento dell'equilibrio territoriale e alla promozione della diversità delle aree rurali; tramandare alle nuove generazioni il proprio valore simbolico, in termini di storia, cultura e tradizioni dei territori di provenienza.

La qualità dei prodotti agroalimentari, dunque, non deve riferirsi semplicemente agli aspetti nutrizionali, sensoriali, organolettici ed igienico-sanitari, tradizionalmente considerati, ma deve essere interpretate in una accezione più estesa che tenga conto degli aspetti ambientali, sociali, culturali e territoriali. Ne deriva, dunque, che con riferimento ai mercati agroalimentari dei paesi avanzati, il concetto di qualità va inteso come un insieme di valori, riferibili ad una quantità di attributi diversi, ciascuno dei quali, assume una maggiore 0 minore importanza a seconda del tipo di prodotto e del segmento, o della nicchia, di mercato al quale il prodotto è destinato (Carbone, 2006). L'insieme di questi valori è riconducibile ad una domanda di consumo sempre più critica, esigente e diversificata che costituisce il motore fondamentale di innovazione sostenibile nel settore agroalimentare (Esposti, 2005).

Partendo da tale assunto, ed in considerazione delle recenti tendenze della domanda alimentare, è possibile identificare alcune direttrici di sviluppo della domanda di qualità espressa dal consumatore, capaci di generare fermento innovativo nel settore agro-alimentare: Healthy living, intesa come l'affermazione di uno stile di vita salutistico; Convenience as a way of life ed Sympathetic food production che si riferisce al modo in cui gli alimenti vengono prodotti e alle tecnologie di produzione utilizzate.

Con riferimento alla prima tendenza, indubbiamente, l'incremento delle aspettative di vita, gli aumenti dei costi sanitari legati ad una maggiore insorgenza di patologie correlabili ad errate abitudini alimentari, la ricerca di migliori standard di qualità, hanno determinato un significativo cambiamento nella domanda alimentare sempre più orientata verso prodotti con forte connotato salutistico (Banterle e Cavaliere, 2009; Carbone, 2006; Verbeke, 2006). ${ }^{5}$

Di conseguenza si sono affermate nell'industria alimentare la sperimentazione e l'utilizzo di tecnologie innovative mirate alla realizzazione di prodotti specifici quali prodotti light, dietetici e functional foods, che rappresentano oggi un segmento di mercato in rapidissima evoluzione, sia nel contesto internazionale che nazionale (Bech-Larsen e Scholderer, 2007; Verbeke, 2006; Sirò et al., 2008). ${ }^{6}$

${ }^{4}$ Nel Libro Verde sulla qualità del 2008, viene ribadito come il perseguimento della qualità rappresenti l'arma più potente di cui dispongano i produttori comunitari per vincere le sfide commerciali in un mondo sempre più globalizzato e di conseguenza l'UE deve sostenere e promuovere sempre più un'offerta alimentare qualificata (COM (2008) 641).

${ }^{5}$ Tale tendenza trova conferma nell'andamento della domanda di prodotti salutistici che, con particolare riferimento al contesto nazionale, ha registrato, nel corso del quinquennio 2002/06, il maggior tasso di crescita dei volumi di acquisto. Inoltre, nel 2007 circa il 30\% delle famiglie ha dichiarato di consumare functional foods.

${ }^{6}$ Dati recenti dell'industria alimentare mostrano che oltre 4000 prodotti sono stati riformulati riducendo o eliminando $i$ grassi saturi, colesterolo, sale, ed acidi grassi per rispondere alle domande dei consumatori nazionali; pertanto non meraviglia che stime attuali attribuiscano agli alimenti funzionali circa il 17\% del totale delle vendite alimentari del paese. 
Con riferimento alla seconda direttrice, la maggiore partecipazione femminile al mercato del lavoro e l'evoluzione delle caratteristiche della famiglia, hanno contribuito ad affermare un nuovo tipo di domanda time saving orientata, cioè ad alimenti che permettano al consumatore di soddisfare le esigenze primarie e contemporaneamente, di risparmiare tempo per gli acquisti, la preparazione e il consumo di cibo. Tale orientamento ha favorito l'intensificarsi degli investimenti in ricerca ed innovazione nell'industria alimentare a supporto dei cosiddetti convenience food, ossia prodotti che consentono un notevole risparmio di tempo nelle varie fasi del processo di acquisto, preparazione e consumo. Infine, con riferimento alla terza direttrice, si sono affermati, negli ultimi anni modelli di consumo che hanno come denominatore comune la modalità di scelta del bene, che prende in considerazione gli effetti sociali e ambientali del ciclo di vita del prodotto, discostandosi dal semplice consumo utilitaristico, poiché attribuiscono valore ad altre componenti del prodotto con particolare riferimento alle conseguenze 0 gli effetti della sua produzione e commercializzazione sull'ambiente e sul benessere collettivo (Briamonte e Hinna, 2008). In questo senso, gli sforzi innovativi nel settore agroalimentare si sono rivolti allidentificazione di prodotti e di processi capaci di combinare gli aspetti economici, ambientali e sociali della sostenibilità. Gli aspetti ambientali riguardano la salvaguardia dell'ambiente naturale, la preservazione della biodiversità e del benessere degli animali. Esempi significativi in tal senso sono ravisabili non solo nella crescente diffusione dei prodotti derivanti da agricoltura biologica 0 da lotta integrata, di prodotti eco-friendly e certificati, ma anche dalla recente affermazione delle diverse forme di filiera corta, tra le quali è possibile citare, ad esempio i Farmers' market, oppure le forme di commercializzazione alternativa quali i Gruppi d'Acquisto Solidali. ${ }^{7}$ Gli aspetti sociali, invece, si riferiscono alla capacità di migliorare le condizioni di benessere umano in termini di sicurezza, salute, nutrizione, istruzione e rispetto delle libertà fondamentali. Si collocano in tale contesto sia quei prodotti o processi, che garantiscono il rispetto dei diritti dei lavoratori e delle minoranze, quali ad esempio i prodotti del Commercio Equo e Solidale e i sistemi di certificazione etica, sia le innovazioni che mirano al miglioramento del benessere in termini di salubrità e sicurezza alimentare, quali ad esempio le certificazioni di rintracciabilità di filiera e di qualità. Al tempo stesso, è possibile collocare in tale ambito le diverse innovazioni che mirano a garantire un maggiore livello di benessere degli animali, tra le quali rientrano ad esempio alcuni schemi di certificazione specifici quali Freedom Food, Neuland e Label Rouge, che però non trovano applicazione in Italia, ed alcune iniziative volontarie di etichettatura (Segrè et al., 2005; Aragrande et al., 2005). ${ }^{8}$ Solo puntando su questa accezione estesa della qualità alimentare, sarà possibile, soprattutto per le piccole e medie imprese che operano nel settore, fronteggiare le sfide sempre più pressanti del mercato e rispondere alle esigenze dei nuovi modelli di consumo responsabile e sostenibile, che si stanno affermando negli ultimi tempi. In questo contesto, anche le strategie di promozione e valorizzazione della qualità, devono essere rilette in una nuova veste, quali attività strategiche ed operative, orientate a migliorare la creazione di valore del prodotto agendo contemporaneamente non solo sul fronte dell'attribuzione del valore da parte del consumatore e della società più in generale, finalizzato alla promozione di forme di consumo consapevoli, ma anche sull'efficacia economica, ambientale e sociale dei processi di produzione da parte del sistema delle imprese.

${ }^{7}$ Allo stato attuale sono presenti, o sono in fase di attivazione, sul territorio nazionale numerose iniziative riconducibili, pur nella diversità di forme e soggetti promotori, ai farmer's market (Covino et al., 2010) mentre con riferimento ai GAS si deve evidenziare come, pur essendo il fenomeno in forte crescita a livello nazionale, vi è una netta prevalenza di gruppi nel Nord Italia rispetto al Meridione.

${ }^{8}$ Nel contesto europeo, sul piano della comunicazione e dell'informazione al consumatore in materia di benessere animale, non vi è allo stato attuale una armonizzazione tra le diverse iniziative intraprese dai diversi paesi. Per rispondere alla duplice esigenza di informare $i$ consumatori e al tempo stesso di armonizzare le diverse iniziative l'Unione Europea nel Piano di Azione 2006-2010 per la protezione e il benessere degli animali, ha previsto la possibilità di introdurre un sistema di certificazione e di etichettatura basato su un comune standard europeo, con l'aspettativa che attraverso la certificazione del benessere animale si possa sviluppare un sistema informativo rivolto ai consumatori.

\section{Marketing orientato alla società ed agroalimen- tare: verso strategie di valorizzazione respon- sabile}

L'attenzione per la salute, il rispetto per l'ambiente e il territorio, l'interesse per i prodotti di qualità sono le preoccupazioni principali della società moderna e queste, come descritto precedentemente, possono essere considerate anche come driver per l'attivazione dei processi innovativi nelle imprese agroalimentari (Commissione Europea, 2008). I percorsi di sviluppo che stanno caratterizzando il settore agroalimentare sono diversi se non opposti: stretto legame alla tradizione e al territorio o agricoltura hi-tech con nuovi prodotti realizzati mediante l'utilizzo di modificazioni genetiche o bio/nanotecnologie. Attorno a questi ultimi, si è sviluppato un dibattito tra favorevoli e contrari ma vi è anche la consapevolezza che si tratta di una delle frontiere più avanzate e promettenti della ricerca e dell'innovazione tecnologica, i cui effetti vanno attentamente valutati in termini di benessere per la società e costi per i diversi ambiti applicativi in cui possono essere utilizzati (Roselli, 2006). L'Unione Europea ha deciso di non vietare la loro produzione 0 introduzione sul mercato ma ha imposto l'etichettatura OGM free, lasciando piena libertà di scelta di acquisto. Il consumatore è tanto libero quanto confuso, è ormai disorientato dalle molteplicità qualitative degli alimenti e questo potrebbe determinare comportamenti di acquisto non razionali. In tale contesto, è necessario un atto di responsabilità delle imprese per aiutare il consumatore ad acquisire e sviluppare conoscenze per maturare scelte consapevoli.

Di conseguenza si delineano nuove strategie imprenditoriali caratterizzate dal superamento dell'esclusività degli interessi economici per favorire e valorizzare il ruolo sociale dell'impresa agroalimentare. Queste ultime, tuttavia, considerate le influenze che con le loro attività possono esercitare sui costumi, sulle attitudini e sulla cultura della società, nel riformulare le proprie strategie competitive sono chiamate a confrontarsi con obiettivi più generali come la sostenibilità dello sviluppo (Cesaretti, 2011). Tutto ciò, sta determinando l'avvento di un nuovo orientamento strategico di impresa che segna il passaggio, prima, dal prodotto al mercato e, poi, dal consumatore alla società complessivamente considerata (Borrelli et al., 2010).

Tuttavia, perseguire un completo orientamento alla società non può manifestarsi nella sola adozione di innovazioni di prodotto, processo 0 organizzative sostenibili ma è necessario che a tal fine le imprese acquisiscano una maggiore consapevolezza delle proprie responsabilità nei processi di sviluppo della società e su di esse definiscano nuove strategie di valorizzazione.

Riteniamo importante concentrarci proprio sulla necessità di approcci innovativi di marketing, in quanto essi sono l'espressione più immediata dei cambiamenti che intercorrono nel rapporto impresasistema di riferimento e contribuisco a ridefinire rapporti con i clienti, i concorrenti, la comunità locale e la società in generale. ${ }^{9}$

In tale ottica, lo sforzo di quanti adottano un orientamento alla società dovrà, dunque, essere quello di sviluppare una strategia di valorizzazione che ponga al centro l'interesse della società, nella consapevolezza che i principi e gli strumenti del marketing possano contribuire in modo determinante a promuovere tale interesse (Scott, 2003; Mitchell, 2010).

L'attenzione ai temi sociali e la valorizzazione dei principi etici, non sono una novità negli approcci teorici del marketing, infatti, soprattutto dagli anni novanta in poi, si sono sviluppate numerose accezioni di quest'ultimo proprio in funzioni di particolari interessi della società.

${ }^{9}$ Secondo Sheth e Sisodia (2005), infatti, il marketing se utilizzato con giudizio può sviluppare e canalizzare le grandi energie di cui dispone un libero sistema di mercato al fine di conseguire il bene dei consumatori, delle imprese e della società nel suo complesso. Impiegato invece senza la necessaria consapevolezza, il marketing può conseguire gli effetti opposti. 
Un primo esempio può essere rappresentato dalla nascita a metà degli anni settanta dell'Ecological marketing, evolutosi poi in Green Marketing, che ha l'obiettivo di fronteggiare le sfide provenienti dalle istanze socio-ambientali proponendo un approccio che interessa tutte le funzioni aziendali al fine di garantire lo sviluppo e la promozione di prodotti e servizi eco-sostenibili che non abbiano impatti negativi sull'ambiente 0 , in un'accezione più ampia, producano effetti positivi su di esso (Henion e Kinnear, 1976).

All'interesse per le problematiche ambientali, alla metà degli anni ottanta, si affianca anche una maggiore sensibilità ai risvolti etici e sociali, determinando lo sviluppo del filone teorico di marketing etico il cui scopo è combinare opportunamente principi morali ed economici nella definizione delle strategie e delle politiche aziendali (Sciarelli, 2006). È nell'ambito di tale approccio che nel 1991 Kotler (2002) introduce il social marketing che a differenza del precedente si concentra sul determinare cambiamenti sociali nell'interesse collettivo attraverso la promozione di idee e comportamenti. L'obiettivo è un maggiore benessere sociale determinato dal superamento di alcuni comportamenti non ritenuti approvabili dalla stessa società 0 che possono arrecare danni alla collettività. Un inquadramento differente è proposto, successivamente, da Lambin, che con l'introduzione del concetto di marketing responsabile fa riferimento ad una strategia capace di realizzare i propri obiettivi economici secondo modalità che preservino o rafforzino il benessere del consumatore e della società. Tale approccio si fonda nella responsabilità che l'impresa, orientata al profitto, si assume nei confronti dell'ambiente in cui opera (Lambin, 1999; Kotler, 1986).

Negli ultimi tempi, la diffusione a tutti i livelli (istituzionale, imprenditoriale, sociale) dell'interesse alla sostenibilità non poteva essere trascurata, infatti, si sta assistendo allo sviluppo del concetto di marketing sostenibile che, a differenza delle accezioni precedenti, segna il superamento dell'interesse per uno specifico aspetto del benessere (ad es. il green marketing) ampliandone i confini a tutta la società. I suoi obiettivi sono individuabili nella riduzione dei conflitti generati tra bisogni privati e bisogni collettivi e nel superamento del divario sociale ed ecologico (Scott, 2003).

Tali approcci teorici del marketing sono di supporto ad un orientamento strategico alla società in quanto permettono di coniugare obiettivi di profitto non solo con le esigenze immediate del singolo consumatore, ma anche con quelle di lungo termine dell'insieme dei consumatori e della società (Baccarani, 2010). In tale ottica, si determina un'evoluzione delle responsabilità d'impresa con un progressivo ampliamento delle stesse.

Per quanto il settore agroalimentare sia tra i più sensibili e responsabili alla realizzazione e preservazione degli interessi della collettività, le sue specificità non consentono una trasposizione diretta degli approcci al marketing appena presentati (Antonelli, 2004). Di conseguenza, si sono sviluppate numerose riflessioni sulla caratterizzazione del marketing agroalimentare e, quindi, su come integrare in esso l'approccio alla società.

Una possibile soluzione è rappresentata dalla combinazione degli strumenti tradizionali del marketing agroalimentare attivandoli secondo l'approccio del marketing sostenibile, ovvero nel rispetto dei principi della sostenibilità. Espressione di tale logica, può essere la definizione di strategie di valorizzazione delle produzioni agroalimentari di qualità ispirate a temi di rilevanza sociale. In tale contesto assume un ruolo centrale il grado di responsabilità maturato dalle imprese. Questa scelta, tra l'altro, oltre a favorire la collettività, rappresenta la modalità operativa più coerente per assicurarsi uno sviluppo competitivo di lungo periodo, considerato che la sostenibilità è ormai considerata una condizione imprescindibile per uno sviluppo duraturo. Possiamo, quindi, parlare di strategie di valorizzazione responsabile intese come la combinazione di strumenti operativi utilizzati nel rispetto dei principi della sostenibilità e finalizzati sia a soddisfare sia ad influenzare i modelli di consumo verso una maggiore consapevolezza. Tali strategie sono finalizzate, quindi, alla promozione di forme di consumo consapevoli e attente all'impegno sociale, ambientale, territoriale delle imprese, ed in termini più generali, alla diffusione di una nuova cultura alimentare orientata ai principi della sostenibilità (Annunziata e Misso, 2011). La definizione di strategie di valorizzazione responsabile non può avvenire sulla base degli obiettivi generali della sostenibilità, ma è necessario individuare macrointeressi sociali legati alle diverse dimensioni dello sviluppo e interconnessi con l'agroalimentare su cui poi intervenire.

Un primo macrointeresse può essere identificato nella salute, intesa come obiettivo sociale che le imprese agroalimentari hanno l'obbligo di rispettare e garantire con l'offerta di prodotti salubri e sicuri e, al tempo stesso, come espressione delle molteplici relazioni esistenti tra i modelli di consumo e il mantenersi sani. Rispetto a queste ultime, gioca un ruolo fondamentale l'informazione del consumatore. Ad esempio, per la diffusione nelle nostre abitudini alimentari di nuovi prodotti, con caratteristiche intrinseche speciali che possono avere effetti mirati sulla salute, diviene fondamentale trasferire conoscenze legate non solo alle proprietà del prodotto ma anche alla ricerca scientifica, per garantire certezza in merito agli effetti salutistici, e all'etichettatura, strumento di sintesi che, se compreso, contribuirà ad una scelta consapevole del consumatore (Malagoli, 2002; Menrad, 2003).

Altra area di interesse è rappresentata dall'ambiente, sia per le relazioni dirette, processo produttivo-impatto sull'ambiente, sia per le interdipendenze esistenti fra ambiente e qualità del prodotto. Infine, considerata la centralità dell'agroalimentare nei processi di sviluppo dei territori e, allo stesso tempo, la valenza dell'unicum territoriale ${ }^{10}$ nell'essere fonte di vantaggio competitivo per i prodotti agroalimentari, un macrointeresse può essere individuato nell'identità culturale. In altri termini, il rispetto per la salute, il mantenimento dell'integrità ambientale e l'identità culturale territoriale saranno le variabili chiave per intercettare la valorizzazione responsabile, ovvero, i punti di contatto tra i diversi perimetri di azione dell'impresa (mercato, società, territorio ed ambiente) che esprimono la sua capacità di stare sul mercato, ed al tempo stesso, di assicurare e promuovere la salute, salvaguardare l'ambiente e custodire l'identità culturale di un territorio (Borrelli et al., 2010).

Per quanto l'attivazione di queste strategie di valorizzazione dipenda dall'impegno dell'impresa al rispetto dei principi della sostenibilità e agli interessi della società, il loro successo e la loro diffusione è strettamente connessa al supporto delle istituzioni e al coinvolgimento attivo dei consumatori. Nello specifico, considerato che le strategie di valorizzazione responsabile mirano a soddisfare interessi sia di carattere pubblico sia di carattere privato diviene strategico riuscire a creare delle sinergie tra le azioni delle imprese responsabili e quelle programmate dalle istituzioni. Queste ultime, tra l'altro, riconoscendo gli effetti positivi di azioni ispirate ai principi della sostenibilità, potrebbero attivare policies a loro supporto. Tali interventi dovrebbero essere finalizzati non solo al sostegno economico delle imprese 0 agli aspetti coercitivi (regolamentazioni, norme, ecc..) ma anche all'adeguamento del sistema di riferimento per favorire le imprese che hanno già intrapreso questo percorso e stimolare quelle che considerano ancora la sostenibilità come un freno al proprio sviluppo. In tal senso, ad esempio, per le imprese orientate alla società, potrebbero essere utile avere a disposizione sul territorio competenze e professionalità qualificate per la definizione di strategie di comunicazione sostenibile di conseguenza diviene importante adeguare il sistema formativo per il loro sviluppo. Mentre per coinvolgere un maggior numero di imprese un'azione necessaria è sicuramente promuovere a tutti i livelli una nuova cultura imprenditoriale fondata sulla responsabilità.

${ }^{10}$ L'unicum territoriale esprime l'essenza del territorio ed è sintesi dei fattori economici, sociali ed ambientali, strettamente interdipendenti tra loro (Bianco e Cesaretti, 2006). 
Altra variabile essenziale per il successo e la diffusione delle strategie di valorizzazione responsabile è l'esistenza di una domanda sostenibile. Se l'orientamento alla società e di conseguenza l'introduzione di strategie di valorizzazione responsabili sono nati proprio per soddisfare i consumatori che richiedevano il rispetto dei loro valori e dei principi della sostenibilità, nel contempo, queste strategie promuovendo modelli di consumo sostenibili e responsabili ${ }^{11}$ finalizzati ad accrescere la consapevolezza della non neutralità dell'atto di acquisto e dell'esistenza di nuovi doveri sociali accanto al semplice perseguimento di utilità e piacere (Briamonte e Giuca, 2010), possono generare un circolo virtuoso per la diffusione dell'orientamento alla società nelle imprese intercettando la domanda dei consumatori responsabili e favorendo il suo sviluppo futuro. In conclusione, attraverso la valorizzazione responsabile le imprese agroalimentari possono riuscire a favorire un cambiamento culturale volontario nella società soddisfacendo interessi economici ma contribuendo a migliorare il benessere degli individui con il loro consenso e la loro collaborazione.

\section{Conclusioni}

L'intensificarsi del processo di globalizzazione e la crisi economica che ha colpito negli ultimi anni i mercati internazionali, impongono, soprattutto alle imprese agroalimentari, la ricerca di nuovi approcci strategici per sopravvivere sul mercato globale. Al tempo stesso, la necessità di garantire la sicurezza e la salubrità dei prodotti offerti, di contribuire alla preservazione delle risorse naturali ma soprattutto, di rispondere alle crescenti preoccupazioni espresse dalla società, richiedono la capacità di sviluppare strategie innovative per valorizzare la qualità offerta. Sulla base di tali premesse il presente lavoro ha inteso offrire alcune riflessioni sul ruolo centrale che l'innovazione riveste oggi, quale motore dello sviluppo sostenibile nel settore agroalimentare in termini economici, ambientali e sociali, dedicando particolare attenzione al contributo che tale fattore può offrire in termini di diversificazione, promozione e valorizzazione della qualità dei prodotti offerti. In particolare, con riferimento a quest'ultimo aspetto, il lavoro ha evidenziato come l'evoluzione subita nel tempo dal concetto di qualità dei prodotti agroalimentari, richieda necessariamente il ricorso ad un insieme sempre più articolato di strategie e strumenti volti alla creazione, alla promozione e alla comunicazione del surplus di valore in essa contenuto. In questo contesto, si inquadrano le strategie di valorizzazione responsabile, quale forma innovativa di comunicazione sostenibile, capace sia di supportare le imprese nell'integrazione dei principi della sostenibilità nei propri processi strategici e operativi, che di influenzare le scelte di acquisto e, di conseguenza, stimolare il consumo responsabile. Il mantenimento e la valorizzazione dei giacimenti ambientali; il rispetto per il benessere e per la salubrità della collettività; l'equità nella remunerazione delle risorse e la salvaguardia delle identità territoriali rappresentano variabili chiave per impostare strategie di valorizzazione responsabile nel settore agroalimentare. Tuttavia, appare fondamentale sottolineare come tali strategie, devono necessariamente mirare alla valorizzazione dell'intero sistema di valori socio-economico-ambientali che concorre alla generazione della qualità dei prodotti agroalimentari, e soprattutto alla comunicazione incisiva di tali valori ai consumatori finali, attivando, in tal modo, un circolo virtuoso di sviluppo sostenibile.

${ }^{11}$ Il consumo responsabile, consapevole, sostenibile abbraccia una modalità di scelta del bene, che prende in considerazione gli effetti sociali e ambientali del ciclo di vita del prodotto e si discosta dal semplice consumo utilitaristico, perché valuta oltre a prezzo e qualità altre componenti del prodotto e, in particolare, le conseguenze o gli effetti della sua produzione e commercializzazione sull'ambiente e sulle persone. L'attenzione del soggetto nell'atto di acquisto è quindi rivolta alle modalità di produzione del bene, alle caratteristiche del soggetto che lo produce e allo smaltimento del prodotto, privilegiando, di conseguenza, processi di produzione e fasi di post-consumo meno inquinanti, che non comportino un depauperamento delle risorse naturali e lavorazioni in cui non siano stati violati diritti umani o norme a tutela delle condizioni di lavoro, quali quelle relative a lavoro nero, impiego di minori, orario, salute e prevenzione (Briamonte e Giuca, 2010).
Le strategie di valorizzazione responsabile, si fondano, dunque, su una visione innovativa delle modalità di comunicare i valori dell'impresa e richiedono una rivisitazione sostanziale dei ruoli e delle responsabilità assunti non solo all'interno dell'impresa stessa, ma anche dalle istituzioni, che sono chiamate a supportare ed incentivare queste nuove modalità di comunicazione, il cui obiettivo ultimo è quello di offrire un contributo alla sostenibilità.

\section{Referenze}

Annunziata A., Misso R., 2011. La valorizzazione sostenibile delle produzioni agroalimentari di qualità. In: A. Annunziata e G.P. Cesaretti (eds.) Strategie e strumenti per la valorizzazione sostenibile delle produzioni agroalimentari di qualità. Franco Angeli Ed., Milano, Italy.

Antonelli G., 2004. Marketing agroalimentare, Specificità e temi di analisi. Franco Angeli Ed., Milano, Italy.

Aragrande M., Segré A., Gentile E., Malorgio G., Giraud Eraud E., Robles Robles R., Halicka E., Loi A., Bruni M., 2005. Food Supply Chain Dynamics and Quality Certification, Comm. Comunità Europee/Joint Research Centre, Institute for Perspective and Technological Studies, Siviglia, Spain. http://foodqualityschemes.jrc.ec.europa.eu/en/documents/ReviewR eport_000.pdf

Baccarani C., 2010. Complessità e intelligenza manageriale. Sinergie 28:97-112.

Banterle A., Cavaliere A., 2009. Determinanti socio-economiche dell'obesità. Agriregionieuropa Anno 5, n.19. http://agriregionieuropa.univpm.it/pdf.php?id_articolo $=520$

Bech-Larsen T., Scholderer J., 2007. Functional foods in Europe: Consumer research, market experiences and regulatory aspects. Trends Food Sci. Tech. 18:231-234.

Bianco M., Cesaretti G.P., 2006. Produzioni Agroalimentari e Territorio - La ricerca dei caratteri distintivi per l'impostazione dei piani di comunicazione. Franco Angeli Ed., Milano, Italy.

Borrelli I.P., Carbone G., Misso R., 2010. La filiera vitivinicola campana tra mercato e società: una strategia di valorizzazione responsabile. Comunicazione presentata al XVIII Convegno Annuale della Società Italiana di Economia Agroalimentare, Venezia, Italy.

Bowersox D.J., Cooper B., Lambert D., Taylor D., 1980. Management in Marketing Channels. McGraw Hill, New York, NY, USA.

Briamonte L., Giuca S., 2010. Comportamenti e consumi socialmente responsabili nel sistema agroalimentare. Inea - Studi e ricerche, Edizioni Scientifiche Italiane, Roma, Italy.

Briamonte L., Hinna L., 2008. La responsabilità sociale delle imprese del sistema agroalimentare. Studi e Ricerche Inea, Roma, Italy.

Carbone A., 2006. La valorizzazione della qualità agroalimentare: diverse strategie a confronto. Agriregionieuropa Anno 2, n. 5. http://agriregionieuropa.univpm.it/dettart.php?id_articolo=113

Cesaretti G.P., 2011. L'approccio della Fondazione Simone Cesaretti alla Sostenibilità dello Sviluppo umano. Rivista di studi sulla sostenibilità n.1/2011. Franco Angeli Ed., Milano, Italy.

Commissione Europea, 2008. LIBRO VERDE sulla qualità dei prodotti agricoli: norme di prodotto, requisiti di produzione e sistemi di qualità, COM(2008) 641. http://europa.eu/legislation_summaries/ agriculture/food/ag0002_it.htm

Covino D., Mariani A., Vastola A., Viganò, E., 2010. Which rules and criteria for a fairer market: a conceptual framework and some evidences about the Italian food supply chain. 119th EAAE Seminar, Capri (Napoli), Italy.

Detre J.D., Johnson A.J., Gray A.W., 2011. Innovativeness and Innovation: Implications for the Renewable Materials Supply 
Chain. Int. Food Agribusiness Manag. Rev. 14:(2). https://www.ifama.org/publications/journal/vol14/cmsdocs/2011005 1_Formatted.pdf

Dosi G., 1982. Technological paradigms and technological trajectories: a suggested interpretation of the determinants and directions of technical change. Res. Policy 11:147-162.

Esposti R., 2005. Cibo e tecnologia: scenari di produzione e consumo alimentare tra tradizione, convenienza e funzione. Agriregionieuropa, anno 1, n.3. http://agriregionieuropa. univpm.it/dettart.php?id_articolo=81

European Commission, 1995. Green Paper on Innovation, COM(95) 688. http://europa.eu/documents/comm/green_papers/pdf/com 95_688_en.pdf

Foray D., 2000. L'économie de la connaissance. La Découverte Ed., Paris, France.

Freeman C., Soete L., 1997. The Economics of Industrial Innovation. Pinter Publ., London, UK.

Henion K.E., Kinnear T.C., 1976. Ecological Marketing. American Marketing Association Ed., Chicago, IL, USA.

Knudson W., Wysocki A., Champagne J., Peterson H.C., 2004. Entrepreneurship and Innovation in the Agri-Food System. Am. J. Agr. Econ. 86:1330-1336.

Kotler P., 1986. Marketing Management. ISEDI Ed., Milano, Italy.

Kotler P., Roberto N., Lee N., 2002. Social Marketing. Improving the quality of life. Sage Publ., Thousand Oaks, CA, USA.

Lambin J.J., 1999. Le marketing stratégique. Du marketing à l'orientation marché, 4ème éd. Ediscience Int., Paris, France.

Malagoli C., 2004. Nutraceutici transgenici: quali opportunità per il consumatore? Rivista di Economia Agro-Alimentare n. 2-3.

Malerba F., 2000. Economia dell'innovazione. Carocci Ed., Roma, Italy.

Menrad K., 2003. Market and marketing of functional food in Europe. J. Food Eng. 56:181-188.
Mitchell R., Wooliscroft B., Higham J., 2010. Sustainable market orientation: A new approach to managing market strategy. J. Macromark. 30:160-170.

Pick J.D., Schneider D., Schnetkamp G., 2001. E-markets. Les nouveaux modèles du B2B. First Editions, Paris, France.

Porter M.E., 2001. Strategia e competizione. Come creare, sostenere e difendere il vantaggio competitivo di imprese e nazioni. Il Sole 24 Ore Libri, Roma, Italy.

Roselli L., Seccia A., Stasi A., 2008. Consumers' attitude towards the new element of evolution of the agro-food system: the introduction of Genetically Modified food. Munich Personal RePEc Archive. http://mpra.ub.uni-muenchen.de/11776/1/MPRA_paper_11776.pdf

Rosenberg N., 1991. Dentro la scatola nera: tecnologia ed economia. Il Mulino ed., bologna, Italy.

Schumpeter J.A., 1942. Capitalism, Socialism and Democracy. Harper and Brothers, New York, NY, USA.

Schumpeter J.A., 1947. The Creative Response in Economic History. J. Econ. Hist. 7:149-159.

Sciarelli S., 2006. Etica e marketing: questioni teoriche e aspetti pratici. Micro \& Macro Marketing 3:335-360.

Scott W.G., 2003. Sostenibilità del marketing e marketing sostenibile. Micro \& Macro Marketing 2:171-182.

Sheth J.N., Sisodia R.S., 2005. A Dangerous Divergence: Marketing and Society. J. Public Policy Mark. 24:160-162.

Sirò I., Kapolna S., Kapolna B., Lugasi A., 2008. Functional food. Product development, marketing and consumer acceptance: a review. Appetite 51:456-467.

Verbeke W., 2006. Functional foods: Consumer willingness to compromise on taste for health? Food Qual. Pref. 17:126-131.

Westgren R.E., 1998. Innovation and Future Directions of Supply Chain Management in US Agri-Food. Can. J. Agr. Econ. 46:519-524. 